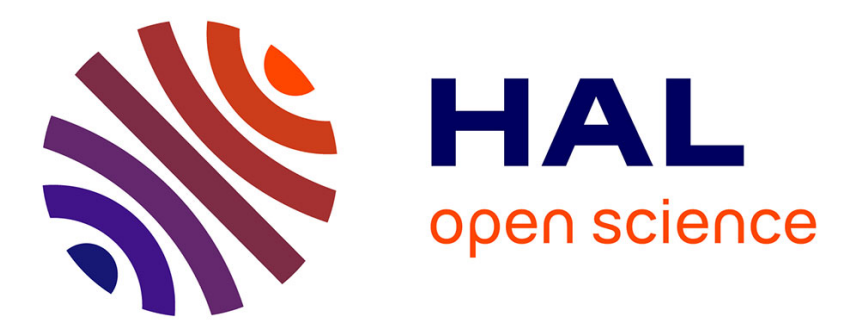

\title{
Probability of relativistic electron trapping by parallel and oblique whistler-mode waves in Earth's radiation belts
}

\author{
A. V. Artemyev, A. A. Vasiliev, D. Mourenas, A. I. Neishtadt, O. V. \\ Agapitov, V. Krasnoselskikh
}

\section{To cite this version:}

A. V. Artemyev, A. A. Vasiliev, D. Mourenas, A. I. Neishtadt, O. V. Agapitov, et al.. Probability of relativistic electron trapping by parallel and oblique whistler-mode waves in Earth's radiation belts. Physics of Plasmas, 2015, 22 (11), 112903 (12 p.). 10.1063/1.4935842 . insu-01255061

\section{HAL Id: insu-01255061 https://hal-insu.archives-ouvertes.fr/insu-01255061}

Submitted on 13 Jan 2016

HAL is a multi-disciplinary open access archive for the deposit and dissemination of scientific research documents, whether they are published or not. The documents may come from teaching and research institutions in France or abroad, or from public or private research centers.
L'archive ouverte pluridisciplinaire HAL, est destinée au dépôt et à la diffusion de documents scientifiques de niveau recherche, publiés ou non, émanant des établissements d'enseignement et de recherche français ou étrangers, des laboratoires publics ou privés.

\section{(1) (1) $\$$}

Distributed under a Creative Commons Attribution - NonCommercial - NoDerivatives| 4.0 


\section{AIP $\left.\right|_{\text {Physics of }}$

\section{Probability of relativistic electron trapping by parallel and oblique whistler-mode waves in Earth's radiation belts}

A. V. Artemyev, A. A. Vasiliev, D. Mourenas, A. I. Neishtadt, O. V. Agapitov, and V. Krasnoselskikh

Citation: Physics of Plasmas 22, 112903 (2015); doi: 10.1063/1.4935842

View online: http://dx.doi.org/10.1063/1.4935842

View Table of Contents: http://scitation.aip.org/content/aip/journal/pop/22/11?ver=pdfcov

Published by the AIP Publishing

\section{Articles you may be interested in}

Stability of relativistic electron trapping by strong whistler or electromagnetic ion cyclotron waves

Phys. Plasmas 22, 082901 (2015); 10.1063/1.4927774

Nonlinear electron acceleration by oblique whistler waves: Landau resonance vs. cyclotron resonance

Phys. Plasmas 20, 122901 (2013); 10.1063/1.4836595

Relativistic electron acceleration by oblique whistler waves

Phys. Plasmas 20, 112902 (2013); 10.1063/1.4831965

Computer simulations of relativistic whistler-mode wave-particle interactions

Phys. Plasmas 11, 3530 (2004); 10.1063/1.1757457

Pitch-angle diffusion of relativistic electrons due to resonant interactions with whistler waves

Phys. Plasmas 6, 4597 (1999); 10.1063/1.873747

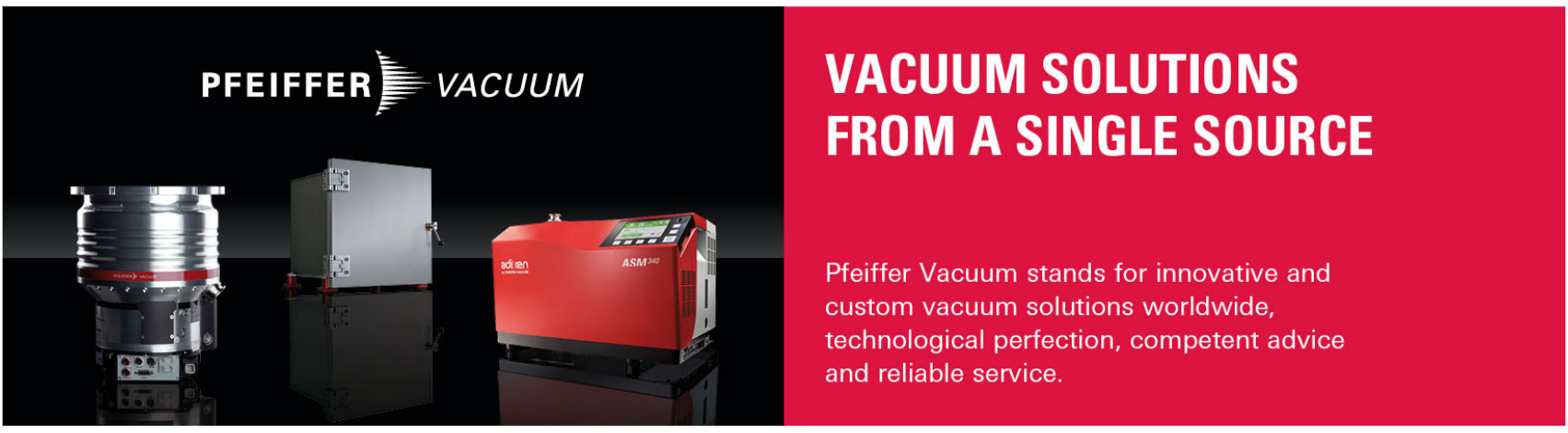




\title{
Probability of relativistic electron trapping by parallel and oblique whistler-mode waves in Earth's radiation belts
}

\author{
A. V. Artemyev, ${ }^{1, \text { a) }}$ A. A. Vasiliev, ${ }^{1}$ D. Mourenas, ${ }^{2}$ A. I. Neishtadt, ${ }^{1, b)}$ O. V. Agapitov, ${ }^{3, c)}$ \\ and V. Krasnoselskikh ${ }^{2}$ \\ ${ }^{1}$ Space Research Institute, RAS, Moscow, Russia, 117997 \\ ${ }^{2}$ LPC2E/CNRS-University of Orleans, 3A, Avenue de la Recherche Scientifique, F-45071 Orleans Cedex, \\ France \\ ${ }^{3}$ Space Sciences Laboratory, University of California, Berkeley, California 94720, USA
}

(Received 13 September 2015; accepted 30 October 2015; published online 17 November 2015)

\begin{abstract}
We investigate electron trapping by high-amplitude whistler-mode waves propagating at small as well as large angles relative to geomagnetic field lines. The inhomogeneity of the background magnetic field can result in an effective acceleration of trapped particles. Here, we derive useful analytical expressions for the probability of electron trapping by both parallel and oblique waves, paving the way for a full analytical description of trapping effects on the particle distribution. Numerical integrations of particle trajectories allow to demonstrate the accuracy of the derived analytical estimates. For realistic wave amplitudes, the levels of probabilities of trapping are generally comparable for oblique and parallel waves, but they turn out to be most efficient over complementary energy ranges. Trapping acceleration of $<100 \mathrm{keV}$ electrons is mainly provided by oblique waves, while parallel waves are responsible for the trapping acceleration of $>100 \mathrm{keV}$ electrons. (C) 2015 AIP Publishing LLC. [http://dx.doi.org/10.1063/1.4935842]
\end{abstract}

\section{INTRODUCTION}

Electron resonant acceleration by whistler-mode waves is believed to play the most important role in the formation of relativistic and ultra-relativistic electron populations in the heart of planetary radiation belts. ${ }^{37,40,42,58,64,67}$ Multiple spacecraft observations of intense emissions of parallel (relative to the background magnetic field) whistler-mode waves during geomagnetic storms and substorms ${ }^{1,33,38}$ have led to the suggestion that these parallel waves could be responsible for almost all the recorded variations of electron fluxes. In addition, oblique wave damping due to Landau resonance with a dense population of suprathermal electrons ${ }^{20,36}$ often sensibly decreases the amplitudes of oblique whistler-mode waves along their propagation.

However, recent data from various satellites in the Earth's inner magnetosphere $2,19,21,34$ indicate that a significant population of high-amplitude and oblique (almost electrostatic) whistler-mode waves is actually present there. Moreover, comprehensive statistics of whistler-mode waves show that even oblique waves with very small magnetic field amplitudes can contribute significantly to resonant waveparticle interactions because of the noticeable portion of wave energy stored in the wave electric field. ${ }^{9}$ This raises an important, as yet unanswered question about the actual relative contributions of oblique and parallel whistler-mode waves in electron acceleration and scattering.

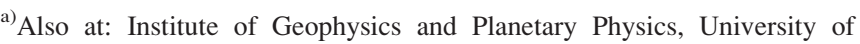
California, Los Angeles, California, USA, CA 90095. Electronic mail: ante0226@gmail.com

b) Also at Department of Mathematical Sciences, Loughborough University, Loughborough LE11 3TU, United Kingdom.

${ }^{c)}$ Also at National Taras Shevchenko University of Kiev, Kiev, Ukraine.
}

The framework of the quasi-linear theory is generally applicable for small to moderate wave amplitudes. ${ }^{54,62}$ In this regard, it has been shown that parallel whistler-mode waves are more intense during high geomagnetic activity periods, while on average, less intense oblique whistlermode waves seem to be responsible for electron acceleration and scattering mainly during relatively quiet conditions 8,39 during which electron quasi-linear pitch-angle diffusion can be considerably enhanced by resonant interaction with such oblique waves. $^{35}$

Nevertheless, significant portions of the observed nonGaussian amplitude distributions of parallel and oblique waves do correspond to very high amplitudes. ${ }^{2,19,21,34,63,66}$ When propagating through background plasma and magnetic field which are both inhomogeneous, such high-amplitude waves can interact with electrons in the nonlinear regime $25,31,48$ when electron trajectories depend significantly on the wave field over time intervals long enough. ${ }^{13,15}$ Such nonlinear interactions include particle trapping and nonlinear scattering (also called phase bunching). ${ }^{56}$ Both effects have been investigated for parallel ${ }^{3,5,17,23,50,62,65}$ and oblique waves. $^{7,14,57,59}$ Although nonlinear scattering is a more widespread effect than particle trapping, the latter can nevertheless be responsible for the rapid acceleration of charged particles ${ }^{17,22,26}$ up to very high energy. Moreover, for realistic wave amplitudes, the net effect of trapping can become more important than both quasi-linear and nonlinear scattering. ${ }^{12,13}$ However, existing analytical estimates and numerical simulations do not allow comprehensive comparisons of the respective efficiencies of parallel and oblique waves in the nonlinear trapping of relativistic electrons under realistic conditions. Answering this important question is the main goal of the present investigation, which is performed following a unified approach based on the Hamiltonian formalism. 
The paper is organized in the following way. First of all, we describe the wave model, i.e., we derive approximate distributions of wave electromagnetic field for two cases: parallel wave propagation and very oblique wave propagation. Then, we use the Hamiltonian approach to provide an invariantbased description of relativistic electron motion in a dipolar magnetic field under the influence of the electromagnetic fields of the considered whistler-mode waves. Such a description is especially appropriate when one wishes to examine phase space fluxes of particles in cyclotron or Landau resonance with the waves. It will allow us to derive analytical expressions for both the probabilities of electron trapping and the corresponding changes in particle energy and pitch-angle. Section V of this paper is devoted to a detailed analysis of these essential, characteristic quantities in realistic conditions corresponding to the Earth's Van Allen radiation belts. A comparison between results obtained for parallel or oblique waves will allow us to address the problem of the relative importance of these two types of waves for electron acceleration.

Note that we consider here a simplified model of background magnetic field (i.e., the curvature-free dipole model proposed in Ref. 14) in which the main magnetic field component depends mainly on the field-aligned coordinate $z$. Although, the effects of curvature of magnetic field lines can be included into the Hamiltonian description of wave-particle resonant interaction, ${ }^{56}$ it would significantly complicate analytical estimates and is therefore left for future works.

\section{WAVE MODEL}

In this paper, we consider parallel and oblique whistlermode waves propagating at a wave normal angle $\theta$ (the angle between the background magnetic field and the wave vector $\mathbf{k}$ ) close to the resonance cone angle $\theta_{r} \approx \arccos \left(\omega / \Omega_{c e}\right)$, where wave frequency $\omega \gg \sqrt{\Omega_{c i} \Omega_{c e}}$ and $\Omega_{c e}, \Omega_{c i}$ are electron and ion gyrofrequencies (i.e., $\theta<88.7^{\circ}$ ). For such waves, we can use the simplified dispersion relation ${ }^{27}$

$$
\omega=\frac{\Omega_{c e} \cos \theta}{1+\left(\Omega_{p e} / k c\right)^{2}},
$$

where $\Omega_{p e}$ is the plasma frequency. The wave vector is lying in the $(x, z)$ plane: $\mathbf{k}=k \sin \theta \mathbf{e}_{x}+k \cos \theta \mathbf{e}_{z}$. Throughout this paper, we consider a constant plasma density $\left(\Omega_{p e} \approx\right.$ const $)$ for the magnetic latitude range $|\lambda|<37^{\circ}$ (the main range of wave-particle resonant interaction), while a more realistic model described in Ref. 24 gives $\Omega_{p e} \sim \cos ^{-5 / 2} \lambda$ in the Earth's outer radiation belt. While this approximation allows us to simplify the final expressions and calculations, it is worth emphasizing that the general equations in Appendix B are derived without any assumption about $\Omega_{p e}$. Estimates of the effect on nonlinear wave-particle interaction of a variation of $\Omega_{p e}$ along magnetic field lines can be found in Refs. 2 and 62. The equatorial value of $\Omega_{p e}$ is determined from an empirical model of plasma density as a function of the McIlwain's parameter $L$ (see Ref. 55).

To describe the distribution of the wave electromagnetic field, we use three components of vector potential $\mathbf{A}=A_{x} \mathbf{e}_{x}$ $+A_{y} \mathbf{e}_{y}+A_{z} \mathbf{e}_{z}$ and scalar potential $\varphi$. The Coulomb gauge gives the relation between two components of $\mathbf{A}: A_{z}=-A_{x} \tan \theta$. Thus, the three components of wave electric field and three components of wave magnetic field can be written as

$$
\begin{aligned}
c E_{x} & =i \omega A_{x}-i k \sin \theta \varphi, \quad c E_{y}=i \omega A_{y}, \\
E_{z} & =-\frac{i \omega}{c} \tan \theta A_{x}-i k \cos \theta \varphi \\
B_{x} & =-i k \cos \theta A_{y}, \quad B_{y}=i \cos ^{-1} \theta k A_{x}, \quad B_{z}=i \sin \theta A_{y} .
\end{aligned}
$$

The relation between components of electromagnetic field ${ }^{61}$ provides the following relations between $A_{x}, A_{y}$, and $\varphi$ :

$$
\begin{aligned}
A_{x} & =i \frac{N^{2}-S}{D} \frac{P \cos ^{2} \theta}{N^{2} \sin ^{2} \theta-P} A_{y} \\
\varphi & =i \frac{N^{2}-S}{D} \frac{N^{2}-P}{N^{2} \sin ^{2} \theta-P} \frac{\sin \theta}{N} A_{y},
\end{aligned}
$$

where $N=k c / \omega$ is the wave refractive index, while $P$, $S=(R+L) / 2, D=(R-L) / 2, R$, and $L$ are Stix coefficients. ${ }^{60}$ Thus, if we introduce $A_{y}=A_{w} \sin \phi=A_{w} \Re\left(-i e^{i \phi}\right)$ (where $\phi$ is the wave phase and $A_{w}$ is amplitude of $A_{y}$ ), then we get

$$
\begin{aligned}
A_{x} & =A_{w} \frac{N^{2}-S}{D} \frac{P \cos ^{2} \theta}{N^{2} \sin ^{2} \theta-P} \cos \phi \\
\varphi & =A_{w} \frac{N^{2}-S}{D} \frac{N^{2}-P}{N^{2} \sin ^{2} \theta-P} \frac{\sin \theta}{N} \cos \phi .
\end{aligned}
$$

The wave phase $\phi$ can be written as

$$
\phi=\int^{z} k_{z}\left(z^{\prime}\right) d z^{\prime}+k_{x} x-\omega t+\phi_{0},
$$

where $k_{z}=k \cos \theta, k_{x}=k \sin \theta$, and $\phi_{0}$ is the initial value of the wave phase.

Let us consider separately two cases: a parallel wave with $\theta=0$ and an oblique wave with $\theta \in\left[\theta_{g}, \theta_{r}\right]$, where $\theta_{g}$ is the Gendrin angle $\theta_{g}=\arccos \left(2 \omega / \Omega_{c e}\right)$.

\section{A. Parallel waves}

For parallel waves $\theta=0$, we have $N^{2}=R$ and Eq. (4) takes the form $A_{x}=-A_{w} \cos \phi, \varphi=0$, where we use $R-S=D$. Equation (5) shows that parallel whistler waves are purely electromagnetic $\mathbf{k} \varphi=0$, circular polarized (amplitude of $A_{x}$ is equal to $A_{y}$ amplitude) waves. The corresponding components of the magnetic field are $B_{x}=-k A_{w}$ $\cos \phi, B_{y}=k A_{w} \sin \phi$. Thus, the total magnetic field amplitude averaged over the wave period $\bar{B}_{w}$ is

$$
\bar{B}_{w}=\sqrt{\frac{1}{2 \pi} \int_{0}^{2 \pi} B_{x}^{2} d \phi+\frac{1}{2 \pi} \int_{0}^{2 \pi} B_{y}^{2} d \phi}=k A_{w} .
$$

For parallel waves, the variation of the wave vector $\mathbf{k}=k \mathbf{e}_{z}$ along magnetic field lines can be obtained from Eq. (1): $k c / \Omega_{c e 0}=\omega_{p e} f_{0}(z)$, where $\Omega_{c e 0}$ is an equatorial value of the electron gyrofrequency, $\omega_{p e}=\Omega_{p e} / \Omega_{c e 0}$ and $f_{0}(z)$ is 


$$
f_{0}(z)=\left(\frac{1}{\cos \theta_{r}}-1\right)^{-1 / 2} .
$$

The corresponding wave phase is

$$
\phi=\phi_{0}+\frac{\Omega_{c e 0}}{c}\left(\omega_{p e} \int^{z} f_{0}\left(z^{\prime}\right) d z^{\prime}-\omega_{m} c t\right),
$$

where $\omega_{m}=\omega / \Omega_{c e 0}$.

\section{B. Oblique waves}

For oblique waves, we can omit terms $\sim \cos ^{2} \theta \ll 1$. Thus, Eq. (4) shows that $A_{x} \sim \cos ^{2} \theta \approx 0$, while for $\varphi$ and $A_{z}$, we can write

$$
\begin{aligned}
A_{z} & =-A_{w} \frac{N^{2}-S}{D} \frac{P \sin \theta \cos \theta}{N^{2} \sin ^{2} \theta-P} \cos \phi \\
\varphi & =A_{w} \frac{N^{2}-S}{D} \frac{N^{2}-P}{N^{2} \sin ^{2} \theta-P} \frac{\sin \theta}{N} \cos \phi .
\end{aligned}
$$

Using Eq. (1), we plot factors $a_{1}=\left(N^{2}-S\right) / D, a_{2}=P \sin \theta /$ $\left(N^{2} \sin ^{2} \theta-P\right), a_{3}=\left(N^{2}-P\right) / P$, and $a=a_{1} a_{2}$ in Fig. 1 . Profiles of $a_{1}, a_{2}$, and $a_{3}$ coefficients show that $a_{1}$ contributes significantly to the wave amplitude $A_{z} / A_{w}, \varphi / A_{w}$, while the smallness of $a_{2}$ is responsible for the weak contribution of $A_{z}$ components to the wave magnetic field.

The components of wave magnetic field (2) take the form

$$
\begin{aligned}
& B_{x}=-k A_{w} \cos \theta \cos \phi, \\
& B_{z}=k A_{w} \sin \theta \cos \phi \\
& B_{y}=k A_{w} a \sin \theta \cos \theta \sin \phi .
\end{aligned}
$$

Thus, the total wave magnetic field amplitude averaged over the wave period $\bar{B}_{w}$ is

$$
\bar{B}_{w}=\sqrt{\frac{1}{2 \pi} \int_{0}^{2 \pi} \mathbf{B}^{2} d \phi} \approx \frac{k A_{w}}{\sqrt{2}} \sqrt{1+\frac{a^{2}}{4} \sin ^{2} 2 \theta} .
$$

Fig. 1 shows that we cannot omit the term $\sim a^{2} \sin ^{2}(2 \theta) / 4$, but we can consider that its magnitude is approximately $\approx 1$. Thus, Eq. (11) gives $\bar{B}_{w} \approx k A_{w}$. Let us now compare the contributions of $A_{z}$ and $\varphi$ to the parallel $E_{z}$ component of the electric field. Equation (7) shows that

$$
\frac{c \partial \varphi / \partial z}{\partial A_{z} / \partial t}=\frac{N^{2}-P}{P}=a_{3}
$$

Fig. 1 shows that the term (12) is much larger than one for $\theta$ close enough to $\theta_{r}$. Thus, we can safely omit the contribution of $A_{z}$ to the wave electric field for oblique waves with wave normal angles in this range.

For oblique waves, the variation of the wave vector magnitude $k$ is given by $k c / \Omega_{c e 0}=\omega_{p e}\left(\cos \theta / \cos \theta_{r}-1\right)^{-1 / 2}$, where $\Omega_{c e 0}$ is the equatorial gyrofrequency. We consider waves propagating with the angle $\theta \in\left[\theta_{g}, \theta_{r}\right]\left(\cos \theta=\left(q \omega / \Omega_{c e}\right)\right.$ and parameter $q \in[1,2])$. For such waves, $\cos \theta / \cos \theta_{r}=q$ and $k c / \Omega_{c e 0}=\omega_{p e} / \sqrt{q-1}$. Thus, the magnitude of the wave vector $k c / \Omega_{c e 0}=\omega_{p e} / \sqrt{q-1}$ does not depend on $z$. The corresponding wave phase is

$$
\phi \approx \phi_{0}+\frac{\Omega_{c e 0}}{c}\left(\frac{q \omega_{p e} \omega_{m}}{\sqrt{q-1}} \int^{z} \frac{\Omega_{c e 0} d z^{\prime}}{\Omega_{c e}\left(z^{\prime}\right)}+\frac{\omega_{p e} x}{\sqrt{q-1}}-\omega_{m} c t\right),
$$

where $\omega_{m}=\omega / \Omega_{c e 0}$ and we assume $\sin \theta \approx 1$ for $\omega_{m}<0.35$.

\section{PROBABILITY OF TRAPPING}

To evaluate the effect of trapping on the distribution function of electrons, one should calculate the energy and pitch-angle shifts of the particles corresponding to a given trapping event (already derived in Ref. 5 for parallel waves and in Refs. 7 and 11 for oblique waves), but also the probability $\Pi$ of such trapping events. The probability of electron trapping $\Pi$ can be defined as the ratio of the number of particles trapped by the wave during their first resonant interaction to the total number of particles having passed through the resonance. To evaluate such quantities, it is convenient to use the Hamiltonian framework. A first step then consists of providing an accurate Hamiltonian description of the motion of particles in resonance with parallel and oblique waves. On this basis, it will be possible to estimate the proportion of particles that eventually get trapped and therefore the global effects of trapping on the electron distribution.

Let us consider the motion of relativistic electrons with charge $e$ and rest mass $m_{e}$ in a background magnetic field $B(z)=B_{e q} b(z)$, with a corresponding vector potential $A_{0 y}=x B(z)$, where $b(z)$ varies with $z$ as the amplitude of the dipole field. ${ }^{14}$ In this case, particle motion can be described by the following Hamiltonian:

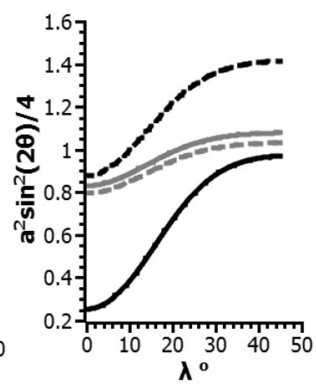

FIG. 1. Profiles of coefficients $a_{1}$ $=\left(N^{2}-S\right) / D, a_{2}=P \sin \theta /\left(N^{2} \sin ^{2} \theta-P\right)$, $a_{3}=\left(N^{2}-P\right) / P$, and $a=a_{1} a_{2}$ along magnetic field lines for several values of the parameter $q=\cos \theta / \cos \theta_{r}$. 


$$
\mathrm{H}=\sqrt{m_{e}^{2} c^{4}+\left(\mathbf{p}+e \mathbf{A}+e A_{0 y} \mathbf{e}_{y}\right)^{2}}-e \varphi,
$$

where $\mathbf{p}$ is the particle momentum ( $p_{y}=$ const and we set $p_{y}=0$ without loss of generality), while $\mathbf{A}$ is the wave vector potential. We introduce dimensionless variables $\mathbf{p} / m_{e} c \rightarrow \mathbf{p}$ and $(x, z) / R_{0} \rightarrow(x, z)$ (where $R_{0}=R_{E} L$ and $R_{0}$ the equatorial distance to the center of the Earth, and $R_{E}$ the radius of the Earth). To keep the Hamiltonian form of the equations, the time should be normalized as $t c / R_{0} \rightarrow t$ (i.e., the unit of dimensionless time is about a quarter of the bounce period of relativistic electrons). We also introduce the dimensionless parameter $\chi=\Omega_{c e 0} R_{0} / c \gg 1$.

We further rewrite the vector potential under the form $A_{w}=u(z) A_{w 0}$, so that the function $u(z)$ alone contains the variation of amplitude $A_{w}$ along the magnetic field lines, while the dimensionless wave amplitude is given by the fixed parameter $b_{w}=e A_{w 0} / m_{e} c^{2}$. For parallel waves, this parameter $b_{w}$ can be written as (see Eqs. (6) and (7)) $b_{w}=e A_{w 0} / m_{e} c^{2}$ $=\bar{B}_{w} / B_{e q} \omega_{p e}$, while for oblique waves, it can be written as $b_{w}=e A_{w 0} / m_{e} c^{2}=\bar{B}_{w} \sqrt{q-1} / B_{e q} \omega_{p e}$, where $B_{e q}$ is the equatorial value of the dipolar magnetic field and $q \in[1,2]$ is a constant.

For both oblique and parallel waves, the function $u(z)$ corresponds mainly to wave amplitude variations caused by wave amplification and damping over the course of interactions with background electron populations: it can be determined from spacecraft observational data (see Fig. 2). For oblique waves, the wave amplitude distribution $u(z)$ has a minimum at the equator. It has been obtained by fitting approximately statistical satellite observations of oblique chorus wave electric fields in the radiation belts (see discussion and explanation in Ref. 7). The magnetic amplitudes of intense parallel waves in the night sector of the inner magnetosphere have usually an opposite distribution with a maximum near the equator and a vanishing level at magnetic latitudes $\lambda>10^{\circ}$ (see Refs. 1, 39, and 53). It corresponds approximately to the size of the equatorial generation region of intense parallel chorus waves, ${ }^{1,52}$ which tend to be damped by suprathermal electrons and/or become more oblique due to refraction as they propagate to higher latitudes. $^{39}$ Moreover, for parallel waves, the function $u(z)$

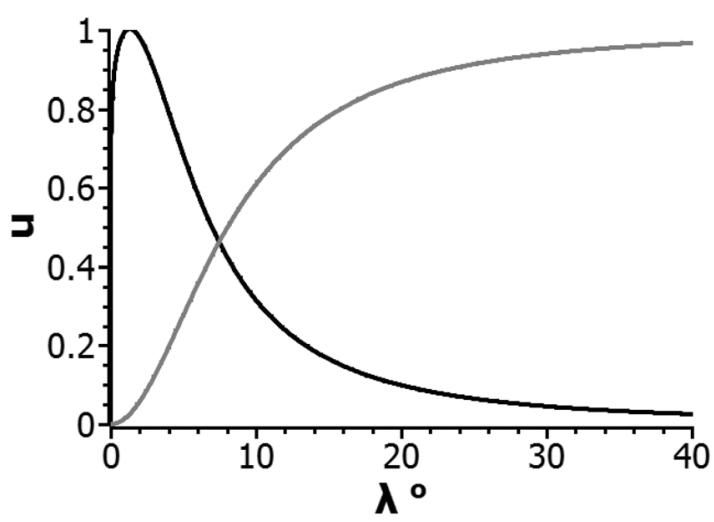

FIG. 2. Profiles of the $u(\lambda)$ function describing the amplitude distribution of oblique (grey) and parallel (black) waves as a function of the magnetic latitude $\lambda$. shown in Fig. 2 should be multiplied by the remaining function $1 / f_{0}(z)=\sqrt{b(z) / \omega_{m}-1}$ (see Eq. (7)) corresponding to the variation of the wave vector.

Finally, expanding the Hamiltonian (14) over the small parameter $b_{w}=e A_{w 0} / m_{e} c^{2} \ll 1$, and making several transformations of variables (see Appendix A for details), we obtain the final form of the Hamiltonian, for both parallel and oblique waves

$$
\begin{aligned}
& \mathrm{H}=\gamma+b_{w} w\left(z, p_{z}, I_{x}\right) \cos \zeta \\
& \gamma=\sqrt{1+p_{z}^{2}+2 I_{x} \chi b},
\end{aligned}
$$

where $I_{x} \chi$ is the magnetic moment (see Eq. (A2)). The effective wave amplitude $w$ and wave phase $\zeta$ are determined separately for parallel and oblique waves. In case of parallel wave propagation and the first cyclotron resonance, we have $w\left(z, p_{z}, I_{x}\right)$ $=-u(z) \sqrt{2 I_{x} \chi b(z)}\left(b(z)-\omega_{m}\right) / \omega_{m} \gamma$ and $\zeta=\phi+\psi$, where $\psi$ is conjugated variable to $I_{x}$ (i.e., $\dot{\psi}=\partial \mathrm{H} / \partial I_{x}$ ) and

$$
\phi \approx \phi_{0}+\chi\left(\int K\left(z^{\prime}\right) d z^{\prime}-\omega_{m} t\right)
$$

with $K(z)=\omega_{\text {pe }} f_{0}(z)$ and $\phi_{0}$ is an initial phase.

For oblique waves and Landau resonance, we have $w$ $=-u(z)\left(\Phi(z) J_{0}(\eta)+\sqrt{2 I_{x} \chi b}\left(d J_{0}(\eta) / d \eta\right)\right)$ and $\zeta=\phi$ from Eq. (16) with $K(z)=q \omega_{p e} \omega_{m} /(b(z) \sqrt{q-1})$. The argument of the Bessel function is $\eta=\sqrt{2 I_{x} \chi b} K(z) / q \omega_{m}$. The function $\Phi(z)$ determines the amplitude of the scalar potential and it is given in Eq. (A1).

The general procedure for the derivation of $\Pi$ has been presented in Refs. 44-47. The probability depends on the rate of evolution of the area $S$ in the phase plane $(\zeta, P)$ surrounded by the separatrix inside which particles have closed trajectories $^{43,57}$ (where $P$ is a variable conjugated to $\zeta$ ). Thus, it can be written as the ratio of the phase flux of particles coming into the region of closed (trapped) trajectories (because of the increase in $S$ ) over the total phase flux passing through the resonance. First applications of this approach to the problem of relativistic electron trapping by whistler-mode waves can be found in Refs. 7 and 11 in the case of oblique waves propagating at the Gendrin angle (see also Supplementary Material in Ref. 12). This procedure essentially requires an expansion of the Hamiltonian (15) around the resonance $\dot{\zeta}=0$. Then, a comparison of phase space fluxes of trapped and transient particles provides the probability of trapping which can be defined as (see Refs. 11 and 29)

$$
\Pi=\frac{2 W}{W+\sqrt{\chi}}
$$

for $W<\sqrt{\chi}$ and $\Pi=1$ otherwise. In Eq. (17), the function $W$ is determined by system parameters and their gradients in phase space (along the resonant trajectory) evaluated at the coordinates of the resonance $z_{R}$ (where $z_{R}$ is a solution of Eq. (15) and $\dot{\zeta}=0$ ). Analytical expressions for $W$ provided in Appendix B for both parallel and oblique whistler-mode waves show that $W$ is proportional to gradients of the function $S\left(z, p_{z}\right)$ (see Eq. (B6)) depending both on magnetic field 

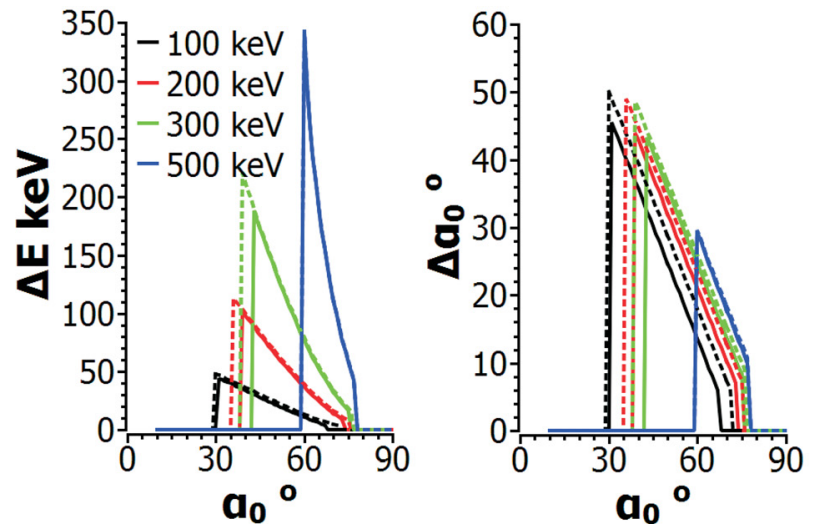

$b(z)$, wavenumber $K(z)$ and effective wave amplitude $w\left(z, p_{z}\right)$. There is no simple analytical expression for $S$ (and for $W$ ), but we can mention one important property of the function $W$. Gradients of $S$ include a term corresponding to $\partial w / \partial z \sim \partial u(z) / \partial z$. Thus, our choice of the function $u(z)$ (see Fig. 2) can influence significantly the final value of the trapping probability $\Pi$. In this study, we use realistic profiles of $u(z)$ for parallel and oblique waves determined from large spacecraft statistics. But the variability of the radiation belts is such that actual profiles may vary sensibly from one event to another. Therefore, for each individual realistic event for which the needed statistical information about $u(z)$ is available, the probability of trapping should be recalculated using the equations from Appendix B.

\section{COMPARISON OF TRAPPING EFFICIENCIES OF PARALLEL AND OBLIQUE WAVES IN THE EARTH'S RADIATION BELTS}

We consider a typical wave frequency $\omega_{m}=0.35$ in the inner magnetosphere, for two $L$-shells $L=4.5$ (with $\omega_{p e}=$ $4, \Omega_{p e}=39.9 \mathrm{kHz}$ ) and $L=6.0$ (with $\omega_{p e}=5.3, \Omega_{p e}=22.5$ $\mathrm{kHz}$ ). In agreement with observational data, the magnetic field amplitude of parallel waves is taken as $B_{w}=1 \mathrm{nT}$ (see, e.g., Refs. 53 and 66), while the magnetic amplitude of

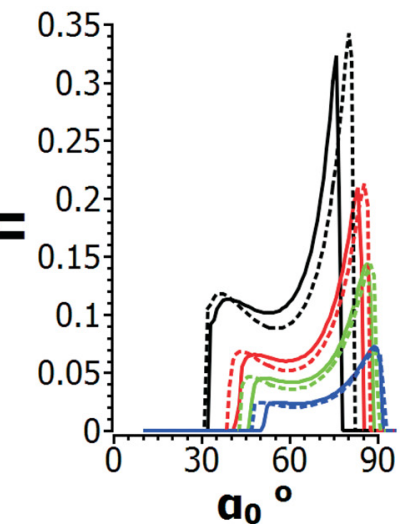

FIG. 3. Distributions of the probability of trapping $\Pi$, as well as energy and pitch-angle jumps are displayed for parallel waves. System parameters are $L=4.5$ (solid curves, $\omega_{p e} \simeq 4$ ) and $L=6.0$ (dotted curves, $\omega_{p e} \simeq 5.3$ ), $\omega_{m}=0.35$ oblique waves is taken as five times smaller than for parallel waves (see, e.g., Refs. 8 and 33).

For both parallel and oblique wave modes, we calculate the probability of particle trapping $\Pi$ for a given equatorial pitch-angle $\alpha_{0}$ and an initial energy $E$, as well as the corresponding jumps in energy $\Delta E$ and pitch-angle $\Delta \alpha_{0}$ due to trapping (see Appendix B for details). Figure 3 shows profiles $\Pi\left(\alpha_{0}\right)$ for parallel waves and four initial particle energies. Trapping is possible for particles with $\alpha_{0}>30^{\circ}$ and trapping acceleration results in a significant increase in the electron energy (energy jumps vary between $50-300 \mathrm{keV}$ ). The probability of trapping $\Pi$ is about $0.1-0.3$ (i.e., up to $30 \%$ of particles get trapped during their first passage through the cyclotron resonance) and it decreases as electron energy increases (at least in the considered energy range).

In Fig. 4, we compare results obtained for parallel and oblique waves. We consider two values of the parameter $q$ : $q=1.05$ corresponding to waves propagating $\sim 1^{\circ}$ below the resonance cone angle, while $q=1.025$ corresponds to waves propagating $\sim 0.5^{\circ}$ from the resonance cone. Such waves with large refractive index are rather common during moderately disturbed periods just outside the plasmasphere., 93 Figure 4 shows that such very oblique waves can resonantly trap only low energy electrons $(E<100 \mathrm{keV})$, while the
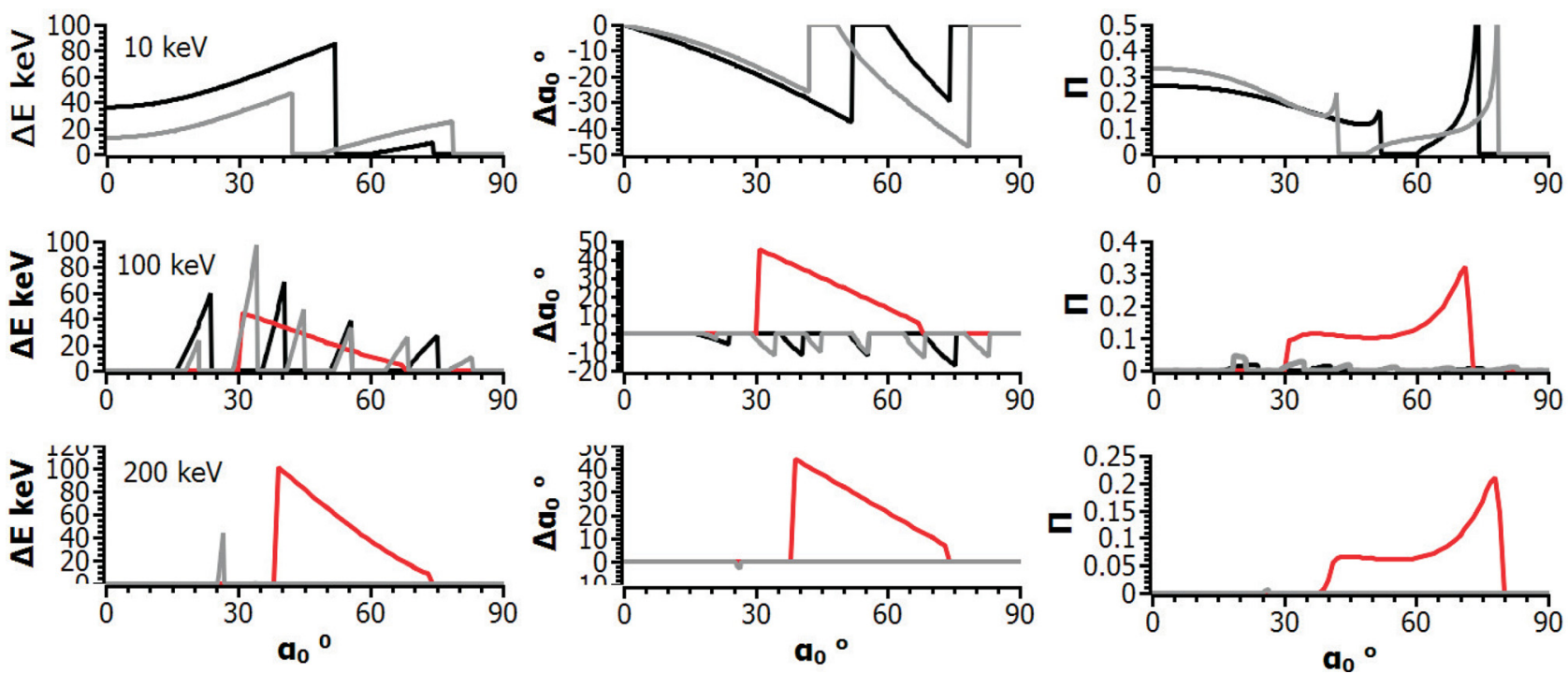

FIG. 4. Comparison of trapping effects for parallel waves (red curve) and for oblique waves with either $q=1.05$ (black curve) or $q=1.025$ (grey curve). Three initial energy values are considered. System parameters are $L=4.5\left(\omega_{p e} \simeq 4\right)$ and $\omega_{m}=0.35$. 
considered parallel waves can only trap electrons with high enough energy $E \geq 100 \mathrm{keV}$. For $100 \mathrm{keV}$ electrons, the amplitude of energy jumps $\Delta E$ is comparable for oblique and parallel waves, but the probability of trapping by parallel waves is much higher. Profiles of energy jumps and probability as functions of $\alpha_{0}$ consist of many peaks corresponding to the maxima of the Bessel function $J_{0}$. Moreover, parallel wave trapping results in an increase in pitch-angle $\Delta \alpha_{0}>0$, while trapping by oblique waves leads to a reduction of pitch-angle (in agreement with previous works, e.g., see Refs. 5 and 7). Interestingly, the probability of trapping is largest for $10 \mathrm{keV}$ electrons and oblique waves. Thus, parallel waves should mainly accelerate electrons of $E>50 \mathrm{keV}$ and shift them to higher pitch-angles where they can remain trapped a long time, while oblique waves accelerate lower energy electrons and simultaneously send them to much lower pitch-angles, which could ultimately hasten the precipitation of one part of them into the atmosphere.

Finally, Fig. 5 shows the maximum (over all equatorial pitch-angles) possible jump of energy $\Delta E$ and the corresponding value of the probability of trapping $\Pi$ for parallel and oblique waves. We consider three values of the wave frequency $\omega_{m}$ and two values of $q$ (i.e., $\theta$ ). For small electron energy $E<50 \mathrm{keV}$, trapping by oblique waves is clearly more effective: both the energy jumps and the corresponding probabilities are larger. But as energy increases, energy jumps $\Delta E$ due to trapping by parallel waves rapidly increase and for $E>200 \mathrm{keV}$ exceed $\Delta E$ due to trapping by oblique waves for all the considered wave frequencies. The probability of trapping by parallel waves with the most effective acceleration (i.e., maximum of $\Delta E$ ) is also larger (to much larger) in general (when it is not equal to zero) than the probability of trapping by oblique waves, demonstrating the likely overall efficiency of this process even in the simultaneous presence of both kinds of waves.

\section{DISCUSSIONS AND CONCLUSIONS}

In this paper, we have focused on electron particle trapping by parallel and oblique whistler-mode waves. However, there exists one additional phenomenon, called particle nonlinear scattering (or phase bunching), which is responsible for small changes of particle energy and pitch-angle. ${ }^{16,28,30,48,51}$ To describe nonlinear scattering, one can use the following Hamiltonian derived by performing an expansion of the equation of motion about the resonance (see, e.g., Refs. 3, 13, and 56):

$$
H_{\phi}=\frac{1}{2} g P^{2}+\mathrm{D} \zeta+\mathrm{B} \cos \zeta
$$

where $(P, \zeta)$ are conjugated variables, and functions $\mathrm{B}(z), \mathrm{D}(z), g(z)$ are given in Eq. (B4). The variable $\zeta$ denotes the wave phase (see Appendix A), while $P$ is equal to $\dot{\zeta} / g$. Thus, there is a direct relationship between $P$ and particle energy $\gamma$ and $I_{x}$. Nonlinear scattering correspond to jumps $\Delta P$, resulting in jumps in energy $\Delta E=m_{e} c^{2} \Delta \gamma$ and pitchangle jump $\Delta \alpha_{0}$. The average value of jumps $\Delta P=P_{0} f_{1}(\mathrm{~A})$ and their variance $\operatorname{Var}(\Delta P)=P_{0}^{2} f_{2}(\mathrm{~A})$ as functions of $\mathrm{B}, \mathrm{D}$, $g$, and $\mathrm{A}=|\mathrm{B} / \mathrm{D}|$ have already been derived for oblique waves in Ref. 13: $P_{0}=\mathrm{B} \sqrt{2 / g \mathrm{D}}$ and the functions $f_{1}(\mathrm{~A}), f_{2}(\mathrm{~A})$ are shown in Fig. 3 of Ref. 13. Previously, a similar approach had been applied in Refs. 4 and 5 to derive energy and pitch-angle jumps due to scattering by parallel whistler-mode waves. Thus, a careful combination of the results obtained in the present paper with previous results from Refs. 4, 5, 11, and 13 should provide all the necessary means for an analytical evaluation of energy and pitch-angle
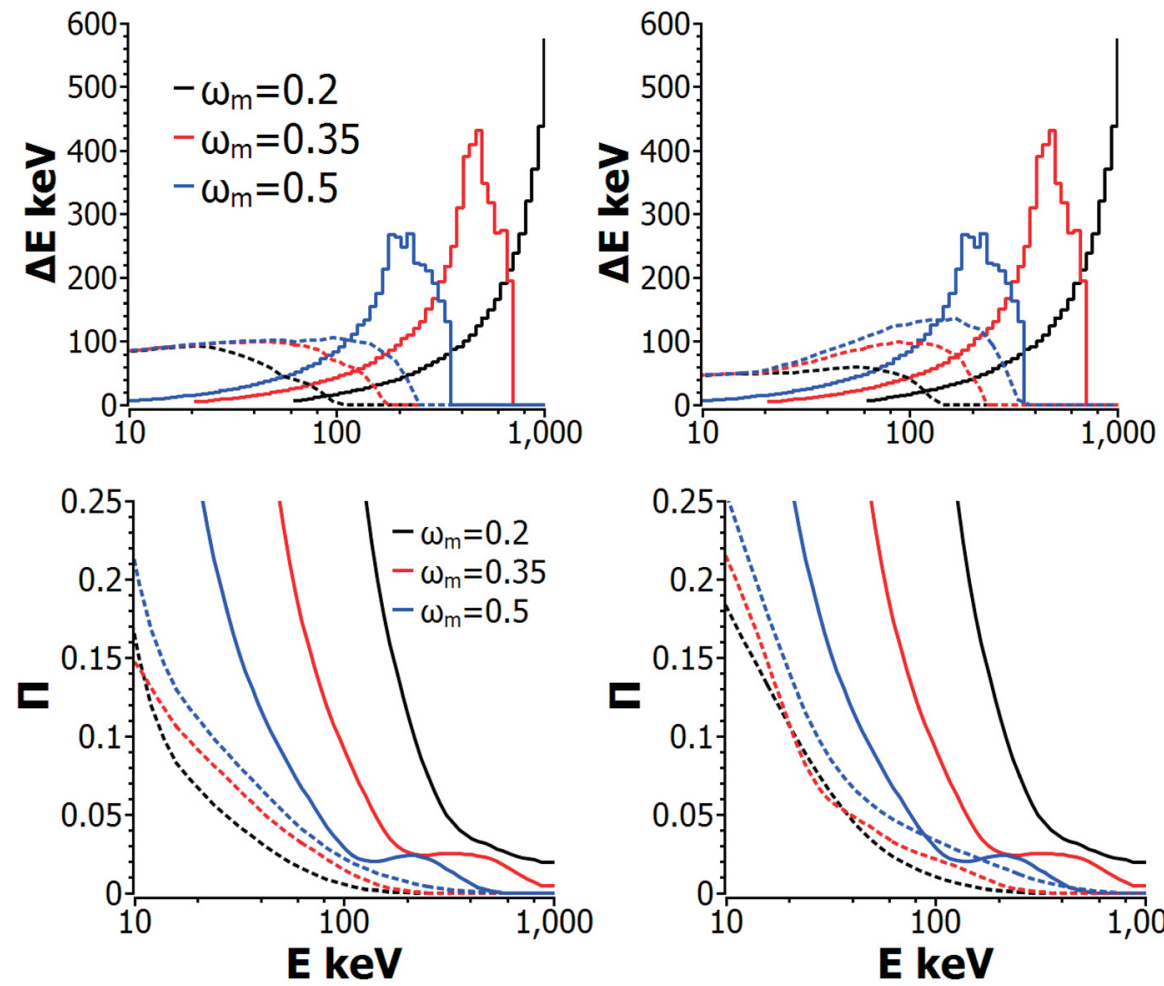

FIG. 5. The maximum jump of energy and the corresponding probability are displayed as a function of the initial electron energy for parallel waves (solid curves) and oblique waves (dashed curves). Left panels correspond to $q=1.05$ and right panels to $q=1.025$. System parameters are $L=4.5\left(\omega_{p e} \simeq 4\right)$ and three values of $\omega_{m}$ are considered. 
variations for both electron trapping and nonlinear scattering by parallel and oblique whistler-mode waves.

Figures 3-5 show that $\geq 100 \mathrm{keV}$ electrons interact with parallel waves much more effectively than with oblique waves. This is due to both the smaller magnetic amplitude of oblique waves and the significant modulation of oblique wave amplitudes by the oscillating Bessel function. While the decrease in the parameter $q$ as $\theta$ gets closer to the resonance cone can substantially increase the wave electric field amplitude, it simultaneously increases the argument of the Bessel function, making the effective wave amplitude more oscillating. As a result, the most effective trapping and acceleration of $\sim 100 \mathrm{keV}$ electrons by oblique waves actually correspond to the Gendrin mode $q=2$ (see Ref. 11). However, for a wave propagating at the Gendrin angle to have a significant parallel electric field, its magnetic field amplitude needs to reach very high levels $\sim 1 \mathrm{nT}$. In this paper, we have considered a more realistic situation in which the magnetic field of oblique waves is five times smaller than for parallel waves. In this case, $q$ should be close enough to 1 to provide electric field amplitudes high enough for nonlinear effects to become important. However, even in this relatively small magnetic amplitude regime, oblique waves turn out to be more effective in trapping and accelerating $<100 \mathrm{keV}$ electrons.

It is also worth noting that additional phenomena could disrupt the stable trapped motion assumed here. For instance, some non-resonant waves of much smaller amplitude may be present in the same space region as the considered waves and can lead to a diffusive destruction of the trapped motion of electrons, ${ }^{10,18}$ while important wave amplitude modulations can lead even more quickly to a particle escape from resonance. ${ }^{10,62}$ The presence of the above effects can reduce trapping-related jumps in electron energy and pitch-angle as compared to the levels calculated here.

In conclusion, we have derived analytical expressions for the probability of electron trapping by parallel and oblique whistler-mode waves. A comparison of these probabilities and the corresponding energy jumps demonstrates that for realistic parallel and oblique wave amplitudes, trapping by parallel waves is more effective for $>100 \mathrm{keV}$ electrons, while for $<100 \mathrm{keV}$ electrons, oblique waves provide a more efficient trapping acceleration. On a global scale, oblique waves might therefore increase the population of $\sim 30-100 \mathrm{keV}$ electrons, while parallel waves could further accelerate these particles up to $\sim \mathrm{MeV}$ energies.

\section{ACKNOWLEDGMENTS}

Work by A.V.A. and A.A.V. was supported by RFBR No. 13-01-00251. Work by A.I.N. was supported by NSh2964.2014.1. Work by O.V.A. was performed under JHU/ APL Contract No. 922613 (RBSP-EFW). A.V.A. is grateful to Dmitry Zimin Dynasty Foundation for support.

\section{APPENDIX A: HAMILTONIAN EQUATIONS OF MOTION}

We start with Hamiltonian (14) and expand it over the small parameter $b_{w}=e A_{w 0} / m_{e} c^{2} \ll 1\left(\right.$ where $\left.A_{w}=u(z) A_{w 0}\right)$

$$
\begin{aligned}
\mathrm{H} & =\gamma+b_{w} u(z) U / \gamma \\
\gamma & =\sqrt{1+p_{z}^{2}+p_{x}^{2}+(\chi x b(z))^{2}} \\
U_{\theta=0} & =\chi x b(z) \sin \phi-p_{x} \cos \phi \\
U_{\theta \sim \theta_{r}} & =\chi x b(z) \sin \phi-\gamma \Phi(z) \cos \phi \\
\Phi(z) & =\frac{N^{2}-S}{D} \frac{N^{2}-P}{N^{2} \sin ^{2} \theta-P} \frac{\sin \theta}{N},
\end{aligned}
$$

where $\mathrm{H} \rightarrow \mathrm{H} / m_{e} c^{2}$ is the dimensionless Hamiltonian, and $\left(x, p_{x}\right),\left(z, p_{z}\right)$ are the pairs of canonically conjugated variables. In Eq. (A1) for oblique waves, function $u(z)$ is shown in Fig. 2, while for parallel waves, $u(z)$ is multiplication of the function shown in Fig. 2 and factor $\sqrt{b / \omega_{m}-1}$. The Hamiltonian (A1) shows that particles rotate around the background magnetic field much faster than they move along field lines $(\chi \gg 1)$. Thus, we can introduce the adiabatic invariant $I_{x}$ corresponding to the averaging over $\left(x, p_{x}\right)$ oscillations $^{32}$

$$
I_{x}=\frac{1}{2 \pi} \oint p_{x} d x=\frac{\gamma^{2}-1-p_{z}^{2}}{2 \chi b(z)}
$$

We write $\left(x, p_{x}\right)$ as functions of the new canonical variables

$$
x=\sqrt{2 I_{x} / \chi b} \sin \psi, \quad p_{x}=\sqrt{2 I_{x} \chi b} \cos \psi,
$$

where phase $\psi$ is conjugated variable to $I_{x}$. It should be noted that due to variation of $b(z)$ with $z$, variable transformation (A3) requires introduction of a new $p_{z}$ which differs from initial $p_{z}$ by the fast oscillating term $(\sim \sin \theta$, see Ref. 32). However, we omit this difference here. With these new variables, the Hamiltonian system (A1) takes a form

$$
\begin{aligned}
\mathrm{H} & =\gamma+b_{w} u U / \gamma \\
\gamma & =\sqrt{1+p_{z}^{2}+2 I_{x} \chi b} \\
U_{\theta=0} & =-\sqrt{2 I_{x} \chi b} \cos (\psi+\phi) \\
U_{\theta \sim \theta_{r}} & =\sqrt{2 I_{x} \chi b} \sin \psi \sin \phi-\gamma \Phi \cos \phi .
\end{aligned}
$$

Thus, for $\psi$, we have the following equation: $\dot{\psi}=\partial \mathrm{H} /$ $\partial I_{x} \approx \chi b / \gamma$. For parallel wave, we can introduce new phase $\zeta_{1}=\phi+\psi$ and write the final form of the Hamiltonian system as

$$
\begin{aligned}
& \mathrm{H}=\gamma-b_{w} \frac{u}{\gamma} \sqrt{2 I_{x} \chi b} \cos \zeta_{1} \\
& \zeta_{1}=\phi_{0}+\chi\left(\int^{z} K\left(z^{\prime}\right) d z^{\prime}-\omega_{m} t\right)+\psi
\end{aligned}
$$

with $K(z)=\omega_{p e} f_{0}(z)$. The corresponding resonance condition $\dot{\zeta}_{1}=0$ represents the first cyclotron resonance $\omega_{p e} f_{0}(z) \dot{z}$ $-\omega_{m}=-b / \gamma$, where $\dot{z}=p_{z} / \gamma$ (see Hamiltonian (A5)).

The wave phase $\phi$ for oblique waves (13) can be written

as

$$
\phi \approx \phi_{0}+\chi\left(\int^{z} K\left(z^{\prime}\right) d z^{\prime}+\frac{\omega_{p e} \sin \psi}{\sqrt{q-1}} \sqrt{\frac{2 I_{x}}{\chi b}}-\omega_{m} t\right)
$$


with $K(z)=q \omega_{p e} \omega_{m} /(\sqrt{q-1} b(z))$. We use two expansions

$$
\begin{aligned}
\cos \phi & =\sum_{n=-\infty}^{\infty} J_{n}(\eta) \cos \left(\zeta_{0}+n \psi\right) \\
\sin \phi \sin \psi & =-\sum_{n=-\infty}^{\infty}\left(d J_{n}(\eta) / d \eta\right) \cos \left(\zeta_{0}+n \psi\right)
\end{aligned}
$$

with $\zeta_{0} \approx \phi_{0}+\chi\left(\int^{z} K\left(z^{\prime}\right) d z^{\prime}-\omega_{m} t\right)$ and $\eta=\sqrt{2 I_{x} b} K / q \omega_{m}$ to rewrite $U_{\theta \sim \theta_{r}}$ from Eq. (A4)

$$
\begin{aligned}
U_{\theta \sim \theta_{r}} & =\sum_{n=-\infty}^{\infty} W_{n} \cos \left(\zeta_{0}+n \psi\right) \\
W_{n} & =-\gamma \Phi J_{n}(\eta)-\sqrt{2 I_{x} \chi b}\left(d J_{n}(\eta) / d \eta\right) .
\end{aligned}
$$

The corresponding resonance condition is $\dot{\zeta}_{0}+n \dot{\psi}=0$ (i.e., $\left.K(z) \dot{z}-\omega_{m}=-n b / \gamma\right)$ and we must take $n=0$ for Landau resonance.

In the case of parallel waves, one can derive from (A4) the following Hamiltonian equations of motion:

$$
\begin{aligned}
\dot{z} & =\frac{\partial \mathrm{H}}{\partial p_{z}}=p_{z} / \gamma \\
\dot{p}_{z} & =-\frac{\partial \mathrm{H}}{\partial z}=-\frac{i_{x}}{\gamma} \frac{\partial b}{\partial z}+b_{w} \chi w K(z) \sin \zeta_{1}+b_{w} \frac{\partial w}{\partial z} \cos \zeta_{1} \\
\dot{i}_{x} & =-\chi \frac{\partial \mathrm{H}}{\partial \psi}=b_{w} \chi w \sin \zeta_{1} \\
\dot{\psi} & =\frac{\partial \mathrm{H}}{\partial I_{x}}=\frac{\chi b}{\gamma}+\frac{b_{w} w}{2 i_{x}} \cos \zeta_{1},
\end{aligned}
$$

where

$$
\begin{aligned}
& i_{x}=\chi I_{x}, \quad \gamma=\sqrt{1+p_{z}^{2}+2 i_{x} b(z)} \\
& w=u(z) \sqrt{b(z) / \omega_{m}-1} \sqrt{2 i_{x} b(z)} / \gamma .
\end{aligned}
$$

For oblique waves, the corresponding Hamiltonian equations of motion are

$$
\begin{aligned}
\dot{z} & =p_{z} / \gamma \\
\dot{p}_{z} & \approx-\frac{i_{x}}{\gamma} \frac{\partial b}{\partial z}+b_{w} \chi w \sin \zeta_{0} \\
w & =-u(z)\left(\Phi(z) J_{0}(\eta)+\sqrt{2 i_{x} b(z)}\left(d J_{0} / d \eta\right)\right),
\end{aligned}
$$

where $i_{x}=$ const; we neglected terms $\sim b_{w} \ll 1$ but kept terms $\sim b_{w} \chi \sim 1$. The above equations are equivalent to the equations of motions derived recently in Ref. 49 .

\section{APPENDIX B: PROBABILITY OF TRAPPING}

In this appendix, we derive the equations for the $W$ function in Eq. (17). We start from the Hamiltonian (15) and perform several changes of variables.

\section{Parallel waves}

First of all, we need to introduce the phase $\zeta$ as a new variable ( $\zeta$ is equal to $\zeta$ from Appendix A). This procedure consists of two steps: introduction of time $t$ as a new variable (with the corresponding conjugated momentum) and change of variables with the introduction of $\zeta$ as a new variable. The first step is technical and well described in Refs. 29, 44, 46, and 47. Thus, we start from the second step and introduce the generating function with term $\zeta I$, where $I$ is the variable conjugate to $\zeta$. We would like to keep the coordinate $z$ and phase $\psi$ as Hamiltonian variables, thus, the second term of the generation function should be $p z+\hat{I}_{x} \psi$, where $p, \hat{I}_{x}$ are new momenta conjugated to $z$ and $\psi$. Therefore, we use the generating function $G_{1}=\zeta I+p z+\hat{I}_{x} \psi$, where $\zeta=\phi+\psi$. This generating function gives $p_{z}=\partial G_{1} / \partial z=p+\xi K(z) I$ and $I_{x}=\partial G_{1} / \partial \psi=\hat{I}_{x}+I$. Thus, we have $p=p_{z}$ $\xi K(z) I, \hat{I}_{x}=I_{x}-I$ (we keep the notation $I_{x}$ for variable $\hat{I}_{x}$ ). Below, we use $I$ and $I_{x}$ instead of $\xi I, \xi I_{x}$. Therefore, the new Hamiltonian has the form

$$
\begin{aligned}
& \mathrm{H}=-\omega_{m} I+\gamma+b_{w} w\left(p, z, I+I_{x}\right) \cos \zeta \\
& \gamma=\sqrt{1+(p+K I)^{2}+2\left(I+I_{x}\right) b .}
\end{aligned}
$$

The Hamiltonian (B1) does not depend on $\psi(\zeta$ is the new variable); thus, $I_{x}$ becomes a constant. The resonance condition $\dot{\zeta}=0$ is

$$
\frac{\partial \mathrm{H}}{\partial \boldsymbol{I}}=-\omega_{m}+\frac{1}{\gamma}(K(p+K I)+b)=0 .
$$

The corresponding value of the resonant $I$ is

$$
I_{R}=\frac{1}{K}\left(v_{\phi} \gamma_{\phi} \sqrt{1-B^{2}-2 B\left(p-K I_{x}\right)}-B-p\right),
$$

where $v_{\phi}=\omega_{m} / K, B=b / K, \gamma_{\phi}=\left(1-v_{\phi}^{2}\right)^{-1 / 2}$. Next, we expand the Hamiltonian (B1) around the resonance $I=I_{R}$ :

$$
\begin{aligned}
\mathrm{H} & =\Lambda+\frac{1}{2} g\left(I-I_{R}\right)^{2}+b_{w} w_{R} \cos \zeta \\
\Lambda(p, z) & =-\omega_{m} I_{R}+\left.\gamma\right|_{I=I_{R}} \\
& =-\omega_{m} I_{R}+\gamma_{R}=\gamma_{\phi}^{-2} \gamma_{R}+v_{\phi}(B+p) \\
g(p, z) & =\left.\frac{\partial^{2} \gamma}{\partial I^{2}}\right|_{I=I_{R}}=\frac{K^{2}}{\gamma_{\phi}^{2} \gamma_{R}} \\
w_{R}(p, z) & =\left.w\left(z_{R}\right)\right|_{I=I_{R}} \\
\gamma_{R} & =\gamma_{\phi} \sqrt{1-B^{2}-2 B\left(p-K I_{x}\right)} .
\end{aligned}
$$

Using the function (B4), we can write the expression for $W$ (see Refs. 11, and 44-47)

$$
W=\frac{1}{4 \pi} \frac{\{S, \Lambda\}}{\left|\left\{\Lambda, I_{R}\right\}\right|},
$$

where $\{.$.$\} is the Poisson bracket for variables (z, p)$ and

$$
\begin{aligned}
S & =2^{3 / 2} \sqrt{D} F(\mathrm{~A}) \\
F(\mathrm{~A}) & =\int_{\zeta_{s}} \sqrt{\zeta_{s}+\mathrm{A} \cos \zeta_{s}-\mathrm{A} \cos \zeta-\zeta} d \zeta \\
\mathrm{B} & =\chi b_{w} w_{R}, \quad \mathrm{D}=\left\{\Lambda, I_{R}\right\} \\
\mathrm{A} & =|B / \mathrm{D}|, \quad \zeta_{s}=\arcsin \left(\mathrm{A}^{-1}\right) .
\end{aligned}
$$


In integral (B6), $\zeta^{*}$ is a solution of equation $\zeta_{s}+\mathrm{A} \cos \zeta_{s}-$ A $\cos \zeta-\zeta=0$ (the profile of the function $F(A)$ can be found, e.g., in Fig. 2 of Ref. 41, see also a description of $F(A)$ properties in Ref. 46).

Making use of the properties of the Poisson bracket, we can write $\mathrm{D}=\left\{\gamma_{R}, I_{R}\right\}$ and

$$
\mathrm{D}=\frac{\gamma_{\phi}^{2}}{K \gamma_{R}}\left(\kappa\left(v_{\phi} \gamma_{R}-B\right)^{2}+\beta B\left(p-K I_{x}\right)\right),
$$

where we introduced the following parameters: $\kappa=(1 / K) \partial K / \partial z, \beta=(1 / b) \partial b / \partial z$.

For the calculation of $W$ in Eq. (B5), one should use the derivatives along the resonance trajectory $p=p(z)$ given by the solution of a system of two equations (B1) with $\mathrm{H}=h$ and (B2)

$$
p=\frac{1}{v_{\phi}^{2}}\left(v_{\phi} h-B+\frac{1}{\gamma_{\phi}} \sqrt{v_{\phi}^{2}+B^{2}-2 B v_{\phi}\left(h-\omega_{m} I_{x}\right)}\right),
$$

where $h$ is the initial value of $-\omega_{m} I+\gamma=\gamma$, corresponding to an initial $I=0$.

To check the derived equations, we numerically integrate particle trajectory described by Eq. (A9) and compare the corresponding results with analytical equations derived in this appendix. Figure 6 shows an example of particle trajectories. The fragments of particle trajectories in the $\left(z, p_{z}\right)$ plane demonstrate the trapped motion. The analytical estimate $\gamma_{R}$ given by Eq. (B4) coincides with numerical results for time periods corresponding to the trapped motion. In the right panels, we show the profiles of area surrounded by the separatrix $S$ (see Eq. (B6)). One can see that the particle escape from the resonance when the area $S$ rapidly drops to small value (actually, the particle escape from the resonance when $S$ approaches its value at the start of trapping). Thus, our analytical equations describe correctly the system: once trapped, particles start moving while conserving the invariant $I_{\phi}=\int P d \zeta$ (this invariant is equal to the value of $S$ at the moment of trapping) and the moment of escape from the resonance corresponds to a decrease in $S$ down to the value equal to $I_{\phi}$ (see a detailed description of this process in Refs. $6,44,46$, and 47).

Fig. 7 shows profiles of analytical probabilities (17) calculated with (B5). The particular, but realistic, distribution of wave intensity $u(z)$ displayed in Fig. 2 has been used. To verify the analytical estimates, we have used test particle simulations: for given pitch-angle and energy, we run a large ensemble of $10^{4}$ particles and determine how many particles become trapped after their first passage through the resonance. Fig. 7 demonstrates that analytical formulas provide an accurate description of the actual probability of trapping. Making use of the conservation of the invariant $I_{\phi}=\int P d \zeta$ for the motion of trapped particles, one can calculate the position of particle escape from the resonance. Thus, the analytical equations provide the energy and pitch-angle jumps of trapped particles (see details of the calculations in Ref. 11). We have also compared the results of analytical estimates against numerical simulations for these jumps in energy and pitch-angle (corresponding to variations taking place between the start of trapping and the escape from resonance). Fig. 7 demonstrates that both energy and pitch-angle variations of the particles are faithfully described by the above analytical expressions.

\section{Oblique waves}

For oblique waves, the function $w$ does not depend on particle momentum $p_{z}$. Moreover, the phase $\zeta$ does not contain $\psi$ and, as a result, the adiabatic invariant $I_{x}$ is conserved ( $\zeta$ is equal to $\zeta_{0}$ from Appendix A). In this case, the final expressions for the probability $\Pi$ of trapping given by Eq. (17) were derived in Ref. 11 and further tested numerically in Ref. 12. The conservation of $I_{x}$ allows us to introduce $i_{x}=\chi I_{x}$ and to rewrite the Hamiltonian (B1) as

$$
\begin{aligned}
& \mathrm{H}=-\omega_{m} I+\gamma+b_{w} w \cos \zeta \\
& \gamma=\sqrt{1+(p+K I)^{2}+2 i_{x} b .}
\end{aligned}
$$

The resonance condition $\dot{\zeta}=0$ gives the corresponding value for the resonant $I$
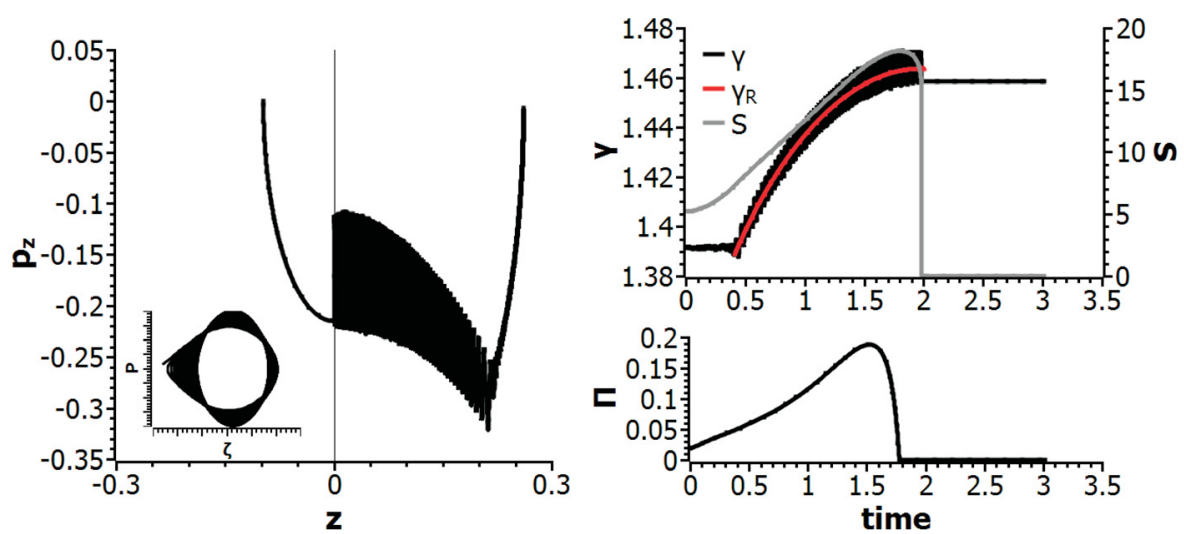

FIG. 6. Trajectory (left panel) and particle energy changes (right panel) for an initially $200 \mathrm{keV}$ electron. System parameters are as follows: $L=4.5$ ( $\omega_{\text {pe }} \simeq 4$ ), the plasma density is taken accordingly to the model presented in Ref. 55, the wave amplitude is $B_{w}=1 \mathrm{nT}$, and the wave frequency is $\omega_{m}=0.35$. Right panels show also a comparison of particle energy $\gamma$ variations obtained from numerical simulations and from analytical estimates, as well as the area surrounded by the separatrix $S$ (see Eq. (B6)). Analytical curves (of $\gamma$ and $I_{R}+I_{x}$ ) are shown in red. Inserted panels display a fragment of particle trajectory in the $(P, \zeta)$ plane corresponding to the trapped motion $(P$ is the momentum conjugated to $\zeta$, see, e.g., Ref. 11). 

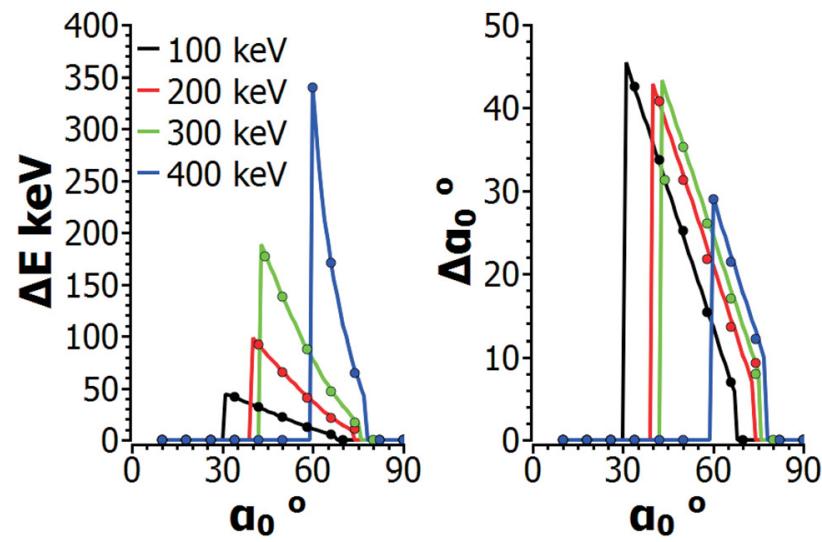

$$
I_{R}=\frac{1}{K}\left(\gamma_{\phi} v_{\phi} \sqrt{1+2 i_{x} b}-p\right) .
$$

Thus, instead of Eq. (B4), we obtain

$$
\Lambda(p, z)=v_{\phi} p+\frac{\sqrt{1+2 i_{x} b}}{\gamma_{\phi}}, \quad g(z)=\frac{K^{2}}{\gamma_{\phi}^{2} \gamma_{R}} .
$$

Equation (B11) provides a simple formula for $W$ :

$$
W=\frac{1}{4 \pi} \frac{v_{\phi}}{\left|\left\{\Lambda, I_{R}\right\}\right|} \frac{\partial S}{\partial z},
$$

where $S$ is given by Eq. (B6), while for $\mathrm{D}=\left\{\Lambda, I_{R}\right\}$ $=K^{-1}\left(\partial \gamma_{R} / \partial z\right)$, we have

$$
\mathrm{D}=\frac{\gamma_{\phi}}{2 K \sqrt{1+2 i_{x} b}}\left(\gamma_{\phi}^{2}\left(1+2 i_{x} b\right) \frac{\partial v_{\phi}^{2}}{\partial z}+2 i_{x} \frac{\partial b}{\partial z}\right) .
$$

Expression (B13) depends only on $z$. Moreover, $\partial v_{\phi} / \partial z$ $=-v_{\phi}(\partial K / \partial z) / K=v_{\phi}(\partial b / \partial z) / b$ (as $K \sim$ const $\left./ b(z)\right)$. Thus, Eq. (B13) takes the form

$$
\left\{\Lambda, I_{R}\right\}=\frac{\gamma_{\phi}^{2}\left(\gamma_{R}^{2} v_{\phi}^{2}+i_{x} b\right)}{K \gamma_{R}} \frac{1}{b} \frac{\partial b}{\partial z} .
$$

The probability of trapping calculated with Eqs. (17) and (B12) has been checked numerically in Refs. 11 and 12 .

${ }^{1}$ O. Agapitov, A. Artemyev, V. Krasnoselskikh, Y. V. Khotyaintsev, D. Mourenas, H. Breuillard, M. Balikhin, and G. Rolland, J. Geophys. Res. 118, 3407-3420, doi:10.1002/jgra.50312 (2013).

${ }^{2}$ O. Agapitov, A. Artemyev, D. Mourenas, V. Krasnoselskikh, J. Bonnell, O. Le Contel, C. M. Cully, and V. Angelopoulos, J. Geophys. Res. 119, 1606-1626, doi:10.1002/2013JA019223 (2014).

${ }^{3}$ J. M. Albert, Phys. Fluids B 5, 2744-2750 (1993).

${ }^{4}$ J. M. Albert, J. Geophys. Res. 105, 21191, doi:10.1029/2000JA000008 (2000).

${ }^{5}$ J. M. Albert, Geophys. Res. Lett. 29, 1275, doi:10.1029/2001GL013941 (2002).

${ }^{6}$ V. I. Arnold, V. V. Kozlov, and A. I. Neishtadt, Mathematical Aspects of Classical and Celestial Mechanics, 3rd ed., Dynamical Systems III. Encyclopedia of Mathematical Sciences (Springer-Verlag, New York, 2006).

${ }^{7}$ A. Artemyev, V. Krasnoselskikh, O. Agapitov, D. Mourenas, and G. Rolland, Phys. Plasmas 19, 122901 (2012).

${ }^{8}$ A. V. Artemyev, O. V. Agapitov, D. Mourenas, V. Krasnoselskikh, and L. M. Zelenyi, Geophys. Res. Lett. 40, 4138-4143, doi:10.1002/grl.50837 (2013).

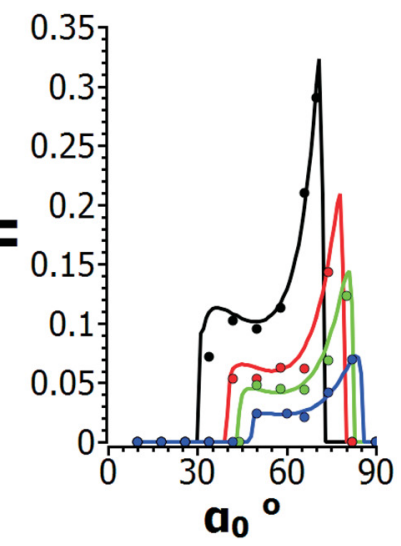

FIG. 7. Probability of trapping $\Pi$, energy, and pitch-angle variations of trapped particles corresponding to the analytical theory (curves) and obtained from test particle simulations (circles) Four values of the initial electron energy (different colors) are considered.

${ }^{9}$ A. V. Artemyev, O. V. Agapitov, D. Mourenas, V. V. Krasnoselskikh, and F. S. Mozer, Nat. Commun. 6, 8143 (2015).

${ }^{10}$ A. V. Artemyev, D. Mourenas, O. V. Agapitov, D. L. Vainchtein, F. S. Mozer, and V. V. Krasnoselskikh, Phys. Plasmas 22, 082901 (2015).

${ }^{11}$ A. V. Artemyev, A. A. Vasiliev, D. Mourenas, O. Agapitov, and V. Krasnoselskikh, Phys. Plasmas 20, 122901 (2013).

${ }^{12}$ A. V. Artemyev, A. A. Vasiliev, D. Mourenas, O. Agapitov, V. Krasnoselskikh, D. Boscher, and G. Rolland, Geophys. Res. Lett. 41, 5727-5733, doi:10.1002/2014GL061380 (2014).

${ }^{13}$ A. V. Artemyev, A. A. Vasiliev, D. Mourenas, O. V. Agapitov, and V. V. Krasnoselskikh, Phys. Plasmas 21(10), 102903 (2014).

${ }^{14}$ T. F. Bell, J. Geophys. Res. 89, 905-918, doi:10.1029/JA089iA02p00905 (1984).

${ }^{15}$ T. F. Bell, J. Geophys. Res. 91, 4365-4379, doi:10.1029/JA091iA04p04365 (1986).

${ }^{16}$ T. F. Bell and U. S. Inan, J. Geophys. Res. 86, 9047-9063, doi:10.1029/ JA086iA11p09047 (1981).

${ }^{17}$ J. Bortnik, R. M. Thorne, and U. S. Inan, Geophys. Res. Lett. 35, L21102, doi:10.1029/2008GL035500 (2008).

${ }^{18}$ A. L. Brinca, J. Geophys. Res. 85, 4711-4714, doi:10.1029/ JA085iA09p04711 (1980).

${ }^{19}$ C. Cattell, J. R. Wygant, K. Goetz, K. Kersten, P. J. Kellogg, T. von Rosenvinge, S. D. Bale, I. Roth, M. Temerin, M. K. Hudson, R. A. Mewaldt, M. Wiedenbeck, M. Maksimovic, R. Ergun, M. Acuna, and C. T. Russell, Geophys. Res. Lett. 35, L01105, doi:10.1029/2007GL032009 (2008).

${ }^{20}$ L. Chen, R. M. Thorne, W. Li, and J. Bortnik, J. Geophys. Res. 118, 1074-1088, doi:10.1029/2012JA018343 (2013).

${ }^{21}$ C. M. Cully, J. W. Bonnell, and R. E. Ergun, Geophys. Res. Lett. 35, L17S16, doi:10.1029/2008GL033643 (2008).

${ }^{22}$ A. G. Demekhov, V. Y. Trakhtengerts, M. Rycroft, and D. Nunn, Geomagn. Aeron. 49, 24-29, doi:10.1134/S0016793209010034 (2009).

${ }^{23}$ A. G. Demekhov, V. Y. Trakhtengerts, M. J. Rycroft, and D. Nunn, Geomagn. Aeron. 46, 711-716, doi:10.1134/S0016793206060053 (2006).

${ }^{24}$ R. E. Denton, K. Takahashi, I. A. Galkin, P. A. Nsumei, X. Huang, B. W. Reinisch, R. R. Anderson, M. K. Sleeper, and W. J. Hughes, J. Geophys. Res. 111, A04213, doi:10.1029/2005JA011414 (2006).

${ }^{25}$ K. B. Dysthe, J. Geophys. Res. 76, 6915-6931, doi:10.1029/ JA076i028p06915 (1971).

${ }^{26}$ N. Furuya, Y. Omura, and D. Summers, J. Geophys. Res. 113, A04224, doi:10.1029/2007JA012478 (2008).

${ }^{27}$ V. L. Ginzburg and A. A. Rukhadze, Waves in Magnetoactive Plasma, 2nd ed. (Nauka, Moscow, 1975).

${ }^{28}$ U. S. Inan and T. F. Bell, Geophys. Res. Lett. 18, 49-52, doi:10.1029/ 90GL02476 (1991).

${ }^{29}$ A. P. Itin, A. I. Neishtadt, and A. A. Vasiliev, Physica D 141(3-4), 281-296 (2000)

${ }^{30}$ V. I. Karpman and D. R. Shkliar, Planet. Space Sci. 25, 395-403 (1977).

${ }^{31}$ V. I. Karpman and D. R. Shklyar, Sov. JETP 35, 500 (1972).

${ }^{32}$ L. D. Landau and E. M. Lifshitz, Vol. 1: Mechanics. Course of Theoretical Physics (Pergamon, Oxford, 1988).

${ }^{33}$ W. Li, J. Bortnik, R. M. Thorne, and V. Angelopoulos, J. Geophys. Res. 116, A12205, doi:10.1029/2011JA017035 (2011).

${ }^{34}$ W. Li, J. Bortnik, R. M. Thorne, C. M. Cully, L. Chen, V. Angelopoulos, Y. Nishimura, J. B. Tao, J. W. Bonnell, and O. Lecontel, J. Geophys. Res. 118, 1461-1471, doi:10.1002/jgra.50176 (2013). 
${ }^{35}$ W. Li, D. Mourenas, A. Artemyev, O. Agapitov, J. Bortnik, J. Albert, R. M. Thorne, B. Ni, C. A. Kletzing, W. S. Kurth, and G. B. Hospodarsky, Geophys. Res. Lett. 41, 6063-6070, doi:10.1002/2014GL061260 (2014).

${ }^{36}$ W. Li, R. M. Thorne, J. Bortnik, Y. Nishimura, V. Angelopoulos, L. Chen, J. P. McFadden, and J. W. Bonnell, J. Geophys. Res. 115, A00J10 (2010).

${ }^{37}$ W. Li, R. M. Thorne, Q. Ma, B. Ni, J. Bortnik, D. N. Baker, H. E. Spence, G. D. Reeves, S. G. Kanekal, J. C. Green, C. A. Kletzing, W. S. Kurth, G. B. Hospodarsky, J. B. Blake, J. F. Fennell, and S. G. Claudepierre, J. Geophys. Res. 119, 4681-4693, doi:10.1002/2014JA019945 (2014).

${ }^{38}$ N. P. Meredith, R. B. Horne, and R. R. Anderson, J. Geophys. Res. 106, 13165-13178, doi:10.1029/2000JA900156 (2001).

${ }^{39}$ D. Mourenas, A. V. Artemyev, O. V. Agapitov, and V. Krasnoselskikh, J. Geophys. Res. 119, 2775-2796, doi:10.1002/2013JA019674 (2014).

${ }^{40}$ D. Mourenas, A. V. Artemyev, O. V. Agapitov, V. Krasnoselskikh, and W. Li, J. Geophys. Res. 119, 9962-9977, doi:10.1002/2014JA020443 (2014).

${ }^{41}$ D. Mourenas, A. V. Artemyev, O. V. Agapitov, V. Krasnoselskikh, and F. S. Mozer, J. Geophys. Res. 120, 3665-3683, doi:10.1002/2015JA021135 (2015).

${ }^{42}$ F. S. Mozer, O. Agapitov, V. Krasnoselskikh, S. Lejosne, G. D. Reeves, and I. Roth, Phys. Rev. Lett. 113(3), 035001 (2014).

${ }^{43}$ A. Neishtadt, J. Appl. Math. Mech. 39, 594-605 (1975).

${ }^{44}$ A. I. Neishtadt and A. A. Vasiliev, Nucl. Instrum. Methods Phys. Res. A 561, 158-165 (2006).

${ }^{45}$ A. Neishtadt, A. Vasiliev, and A. Artemyev, Moscow Math. J. 11(3), 531-545 (2011).

${ }^{46}$ A. I. Neishtadt, Russ. Math. Surv. 69(5), 771 (2014).

${ }^{47}$ A. I. Neishtadt, "Capture into resonance and scattering on resonances in two-frequency systems," Proc. Steklov Inst. Math. 250, 183-203 (2005).

${ }^{48}$ D. Nunn, J. Plasma Phys. 6, 291 (1971).

${ }^{49}$ D. Nunn and Y. Omura, J. Geophys. Res. 120, 2890-2911, doi:10.1002/ 2014JA020898 (2015).

${ }^{50}$ Y. Omura, N. Furuya, and D. Summers, J. Geophys. Res. 112, A06236, doi:10.1029/2006JA012243 (2007).
${ }^{51}$ N. Ryabova and D. Shklyar, Phys. Lett. A 97(5), 194-198 (1983).

${ }^{52}$ O. Santolík, D. A. Gurnett, J. S. Pickett, M. Parrot, and N. CornilleauWehrlin, Planet. Space Sci. 53, 299-305 (2005).

${ }^{53}$ O. Santolík, C. A. Kletzing, W. S. Kurth, G. B. Hospodarsky, and S. R. Bounds, Geophys. Res. Lett. 41, 293-299, doi:10.1002/2013GL058889 (2014).

${ }^{54}$ V. D. Shapiro and R. Z. Sagdeev, Phys. Rep. 283, 49-71 (1997).

${ }^{55}$ B. W. Sheeley, M. B. Moldwin, H. K. Rassoul, and R. R. Anderson, J. Geophys. Res. 106, 25631-25642, doi:10.1029/2000JA000286 (2001).

${ }^{56}$ D. Shklyar and H. Matsumoto, Surv. Geophys. 30, 55-104 (2009).

${ }^{57}$ D. R. Shklyar, Sov. Phys. JETP 53, 1192-1197 (1981).

${ }^{58}$ Y. Y. Shprits, J. D. Menietti, X. Gu, K. C. Kim, and R. B. Horne, J. Geophys. Res. 117, A11216, doi:10.1029/2012JA018031 (2012).

${ }^{59}$ V. V. Solovev and D. R. Shkliar, Sov. Phys. JETP 63, 272-277 (1986).

${ }^{60} \mathrm{~T}$. H. Stix, The Theory of Plasma Waves (McGraw-Hill, New York, 1962).

${ }^{61}$ X. Tao and J. Bortnik, Nonlinear Process. Geophys. 17, 599-604 (2010).

${ }^{62}$ X. Tao, J. Bortnik, R. M. Thorne, J. M. Albert, and W. Li, Geophys. Res. Lett. 39, 6102, doi:10.1029/2012GL051202 (2012).

${ }^{63}$ U. Taubenschuss, Y. V. Khotyaintsev, O. Santolík, A. Vaivads, C. M. Cully, O. L. Contel, and V. Angelopoulos, J. Geophys. Res. 119, 9567-9578, doi:10.1002/2014JA020575 (2014).

${ }^{64}$ R. M. Thorne, W. Li, B. Ni, Q. Ma, J. Bortnik, L. Chen, D. N. Baker, H. E. Spence, G. D. Reeves, M. G. Henderson, C. A. Kletzing, W. S. Kurth, G. B. Hospodarsky, J. B. Blake, J. F. Fennell, S. G. Claudepierre, and S. G. Kanekal, Nature 504, 411-414 (2013).

${ }^{65}$ V. Y. Trakhtengerts, M. J. Rycroft, D. Nunn, and A. G. Demekhov, J. Geophys. Res. 108, 1138, doi:10.1029/2002JA009559 (2003).

${ }^{66}$ L. B. Wilson III, C. A. Cattell, P. J. Kellogg, J. R. Wygant, K. Goetz, A. Breneman, and K. Kersten, Geophys. Res. Lett. 38, 17107, doi:10.1029/ 2011GL048671 (2011).

${ }^{67}$ E. E. Woodfield, R. B. Horne, S. A. Glauert, J. D. Menietti, and Y. Y. Shprits, J. Geophys. Res. 119, 3490-3502, doi:10.1002/2014JA019891 (2014). 